Depression Rating Scale (HAMD21), Clinical Global Impressions Scale (CGI-BP), SF-36, SLICE/Life Scale and the EuroQol. Participants were also asked about age at first major affective episode.

Results: Our data support the existence of three subgroups; early (AAO $<20$, mean $=15.47 \pm 2.7$ ) $46.5 \%$ of participants, intermediate (AAO 20-35, mean $=25.52 \pm 4.4) 43.8 \%$ of participants and late $(\mathrm{AAO}>35$, mean $=46.2 \pm 10.1) 9.7 \%$ of participants. The groups differed significantly in the type of first episode experienced $\left(\chi^{2}=14.88\right.$, df $=1$, $P=0.005)$ such that the early subgroup were more likely to experience a depressive first episode, while the intermediate subgroup were more likely to experience a manic first episode. At enrollment, the early subgroup reported more severe depressive symptoms [HAM-D F $(1,153)=10.20, P=0.007]$. When the early subgroup was compared with the typical subgroup (intermediate and late combined), the early subgroup tended to experience more clinically significant distress as a result of depression (CGI-BP; $\chi^{2}=3.73$, $\mathrm{df}=1, P=0.053)$, were less satisfied with their overall health (SF-36; $\left.\chi^{2}=9.42, \mathrm{df}=4, P=0.051\right)$ and were less able to enjoy recreational activities (SLICE; $\chi^{2}=10.47, \mathrm{df}=4, P=0.033$ ).

Conclusions: Several clinical and functional differences were found between the subgroups based on preliminary data. These differences are important as they can help guide clinical management of this debilitating disorder.

\section{Neuropsychological function in social phobia}

\section{Bourke', R Porter', H Colhoun², F Carter', C Bell'}

'Department of Psychological Medicine, Christchurch School of Medicine and Health Sciences; and ${ }^{2}$ Anxiety Disorders Unit, Canterbury District Health Board, New Zealand

Background: Studies of neuropsychological function in social phobia have focused on attentional processes, memory function, judgment and interpretation biases, but findings have been conflicting. Differences in clinical characteristics or variation in testing paradigms and tasks may account for the inconsistencies. This study aimed to assess several cognitive functions, including verbal declarative memory, working memory, verbal fluency and recognition memory for visuospatial information.

Methods: Thirty adults who met DSM-IV criteria for social phobia and 27 age- and gender-matched healthy controls aged between 18 and 65 years completed neuropsychological testing. Participants were recruited by means of newspaper advertisements. Severity of social phobia was rated using the Liebowitz Social Anxiety Scale. Participants completed a battery of neuropsychological tests including the Rey Auditory
Verbal Learning Test, spatial span, spatial recognition memory, spatial working memory, digit span, and verbal fluency and a verbal memory task comprising nonsense words. The National Adult Reading Test was used to estimate premorbid verbal IQ.

Results: There were no significant group differences on any domain of function, including verbal learning and memory, attention, working memory, verbal fluency, visuospatial functioning or psychomotor speed.

Conclusions: Social phobia was not associated with neuropsychological impairment, but clinical characteristics of the sample may account for this. Patients were highfunctioning individuals with mild to moderate social phobia who had not specifically sought help for social phobia.

\section{The multiscale hypothesis of bipolar disorder}

\section{Breakspear}

School of Psychiatry, University of NSW, and The Black Dog Institute, Sydney, Australia

The brain is characterized by the presence of architectural structures - neurons, minicolumns, cortical columns - across a hierarchy of spatial scales. In addition, the activity of the brain is expressed - through action potentials, EEG oscillations, diurnal rhythms - across a multitude of temporal scales. We propose that bipolar disorder arises as a biological disturbance at a very fine spatial and temporal scale, within transmembrane dynamics, which then cascades across scales to be expressed at the slower scales of symptoms, episodes and ultimately the illness across the life span. This proposal is embedded within a hierarchical model of neocortical activity. Innovative data analysis methods, allowing the investigation of EEG and functional magnetic resonance imaging data from such a multiscale perspective, are presented. We hence propose a set of functional neuroscience experiments that would allow this 'multiscale hypothesis' of bipolar disorder to be tested.

\section{Referential delusions of communication and self-monitoring deficits in psychosis}

\section{S Bucci' ${ }^{1,2}$, T Lewin ${ }^{2}$, A Baker ${ }^{2}$, A Heathcote ${ }^{3}$, M Startup ${ }^{3}$}

Pesychological Assistance Service; ${ }^{2}$ Centre for Mental Health Studies, Newcastle; and ${ }^{3}$ School of Psychology, University of Newcastle, Newcastle, Australia

Background: Although delusions of reference are one of the most frequently occurring symptoms of 